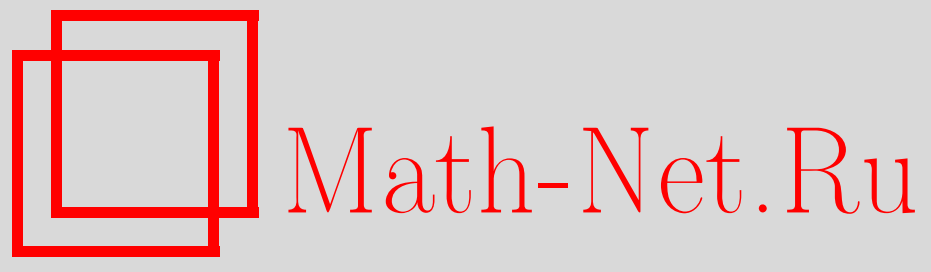

В. А. Кошелев, Компьютерное решение задачи о почти пустых шестиугольниках, Матем. заметки, 2011, том 89, выпуск 3, 477-480

DOI: https://doi.org/10.4213/mzm9053

Использование Общероссийского математического портала Math-Net.Ru подразумевает, что вы прочитали и согласны с пользовательским соглашением http://www . mathnet.ru/rus/agreement

Параметры загрузки:

IP : 3.85 .5 .30

26 апреля 2023 г., 15:12:02

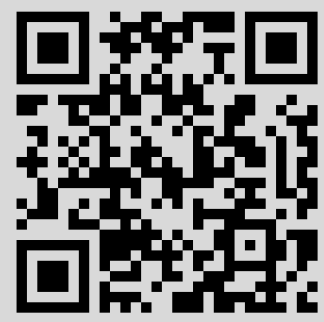




\section{Компьютерное решение задачи о почти пустых шестиугольниках}

\section{В. А. Кошелев}

1. Введение и формулировки результатов. В 1935 г. Эрдеш и Секереш сформулировали следующую проблему (см. [1], [2]).

ПЕРВАЯ ПРОБЛЕМА ЭРДЕША-СЕКЕРЕША. Для любого целого $n \geqslant 3$ найти минимальное положительное число $g(n)$ такое, что из любого множества точек на плоскости, находящегося в общем положении и содержащего, по крайней мере, $g(n)$ точек, можно выбрать подмножество мощности $n$, элементы которого являются вершинами выпуклого п-угольника.

В 1978 г. Эрдеш предложил следующую модификацию первой проблемы (см. [3]).

ВТОРАЯ ПРОБЛЕМА ЭРДЕША-СЕКЕРЕША. Для любого целого $n \geqslant 3$ найти минимальное положительное число $h(n)$ такое, что из любого множества точек $X$ на плоскости, находящегося в общем положении и содержащего, по крайней мере, $h(n)$ точек, можно выбрать подмножество мощности п, элементы которого являются вершинами выпуклого и пустого п-угольника, т.е. этот п-угольник не содержит внутри себя других точек из $X$

Напомним, что множество точек находится в общем положении, если никакие три его элемента не лежат на одной прямой.

Перечисленные проблемы являются классическими в комбинаторной геометрии и теории Рамсея (см. [4]-[7]). Обе они обобщаются следующим образом.

ТРЕТЬЯ ПРОБЛЕМА ЭРДЕША-СЕКЕРЕША. Для любъх целы $n \geqslant 3 u k \geqslant 0$ найти минимальное положительное число $h(n, k)$ такое, что из любого множества точек $\mathscr{X}$ на плоскости, находящегося в общем положении и содержащего, по крайней мере, $h(n, k)$ точек, можно выбрать подмножество мощности $n$, элементы которого являются вершинами выпуклого п-угольника $C$ с условием $|(C \backslash \partial C) \cap \mathscr{X}| \leqslant k$, m.e. этот n-угольник содержит внутри себя не более $k$ других точек из $\mathscr{X}$.

Относительно первой проблемы Эрдеша-Секереша известно (см. [4] и [8]), что

$$
g(3)=3, \quad g(4)=5, \quad g(5)=9, \quad g(6)=17 .
$$

Последний результат был доказан значительно позже остальных благодаря усилиям Секереша, Б. МакКея и Л. Питерса. Они разработали алгоритм, позволяющий осуществить исчерпывающий компьютерный перебор все 17-точечных множеств точек на плоскости для того, чтобы во всех случаях убедиться в наличии выпуклого шестиугольника. За 1500 часов работы компьютера теорема была доказана. Краткое описание алгоритма приведено в следующем разделе.

Для общего случая лучшие верхние и нижние оценки таковы:

$$
2^{n-2}+1 \leqslant g(n) \leqslant\left(\begin{array}{c}
2 n-5 \\
n-3
\end{array}\right)+1 .
$$

Вторая проблема изучена в некотором смысле более глубоко. Для нее доказаны следующие результаты (см. [4] и [9]):

$$
h(3)=3, \quad h(4)=5, \quad h(5)=10, \quad 30 \leqslant h(6) \leqslant 463,
$$

и, наконец, при $n \geqslant 7$ величина $h(n)$ не существует.

Работа выполнена при финансовой поддержке Российского фонда фундаментальных исследований, грант № 09-01-00294. 
Для третьей проблемы, как нетрудно видеть, всегда выполнены неравенства

$$
g(n) \leqslant h(n, k) \leqslant h(n),
$$

если соответствующие величины существуют. Более того,

$$
h(n)=h(n, 0) \geqslant h(n, 1) \geqslant h(n, 2) \geqslant \cdots
$$

и найдется такое $k^{\prime}$, что $h(n, k)=g(n)$ при всех $k \geqslant k^{\prime}$. Для малых значений $n$ очевидны следующие результаты:

$$
h(3, k)=3, \quad h(4, k)=5, \quad h(5,0)=10, \quad h(5, \geqslant 1)=9 .
$$

Последний результат обусловлен тем, что выпуклый пятиугольник с двумя или более точками внутри всегда содержит меньший выпуклый пятиугольник.

Некоторые результаты по третьей проблеме получены в статье Сендова (см. [10]). В этой статье с использованием множества Хортона (см. [11]), за счет которого было доказано несуществование $h(7)$, устанавливается несуществование $h(n, k)$ для определенных $k$ при $n>7$. Аналогичные результаты получены в статье Ныкловой (см. [12]) и, кроме того, там доказано, что $h(6, \geqslant 6)=g(6)$, а также представлен результат $h(6,5)=19$. В доказательстве последнего равенства автором были обнаружены неточности, а указанные ниже теоремы его окончательно опровергают.

Лучшие на данный момент значения $k$, при которых $h(n, k)$ не существует, найдены автором и имеют вид $\left(\begin{array}{c}n-7 \\ (n-7) / 2\end{array}\right)-1$ для нечетных $n$ и $\left(\begin{array}{c}n-8 \\ (n-8) / 2\end{array}\right)-1$ для четных (см. [13], [14]).

Интересной задачей также является нахождение значений $k$, для которых $h(n, k)=g(n)$ и $h(n, k)>g(n)$. Автором было доказано следующее неравенство:

$$
h\left(n,\left(\begin{array}{c}
(n-3) \\
\lceil(n-3) / 2\rceil
\end{array}\right)-\left\lceil\frac{n}{2}\right\rceil\right)>2^{n-2}+1
$$

(см. [13], [14]). В правой части этого неравенства находится $2^{n-2}+1$ вместо $g(n)$. Такая замена была произведена ввиду того, что гипотеза о совпадении этих величин остается недоказанной.

Из новых результатов для фиксированных значений $n$ укажем следующее неравенство:

$$
h(6,1) \leqslant g(7) \leqslant 127
$$

(см. [15]). Впрочем, из доказательства ясно, что оценка 127 является завышенной и может быть значительно улучшена. Используя новый алгоритм за основу которого был взят алгоритм Секереша-МакКея-Питерса (см. [8]) и мощный вычислительный кластер, удалось доказать следующие теоремы.

Tеорема 1. Выполнено точное равенство $h(6,2)=17$.

СлЕДСТВИЕ 1. Выполнено точное равенство $h(6, \geqslant 2)=17$.

Теорема 2. Выполнено точное равенство $h(6,1)=18$.

Благодаря теоремам 1 и 2 , теперь известны все точные значения для $h(n, k)$ при $n \leqslant 6$, кроме единственного $h(6,0)=h(6)$.

Теорему 2 иллюстрирует пример множества из 17 точек (см. рис. 1), которое не содержит ни одного выпуклого шестиугольника не более, чем с одной точкой внутри. При этом, как утверждает теорема 1, это множество обязано содержать выпуклые шестиугольники не более чем с двумя (а в данном случае ровно с двумя) точками внутри. Легко убедиться, что это действительно так. 


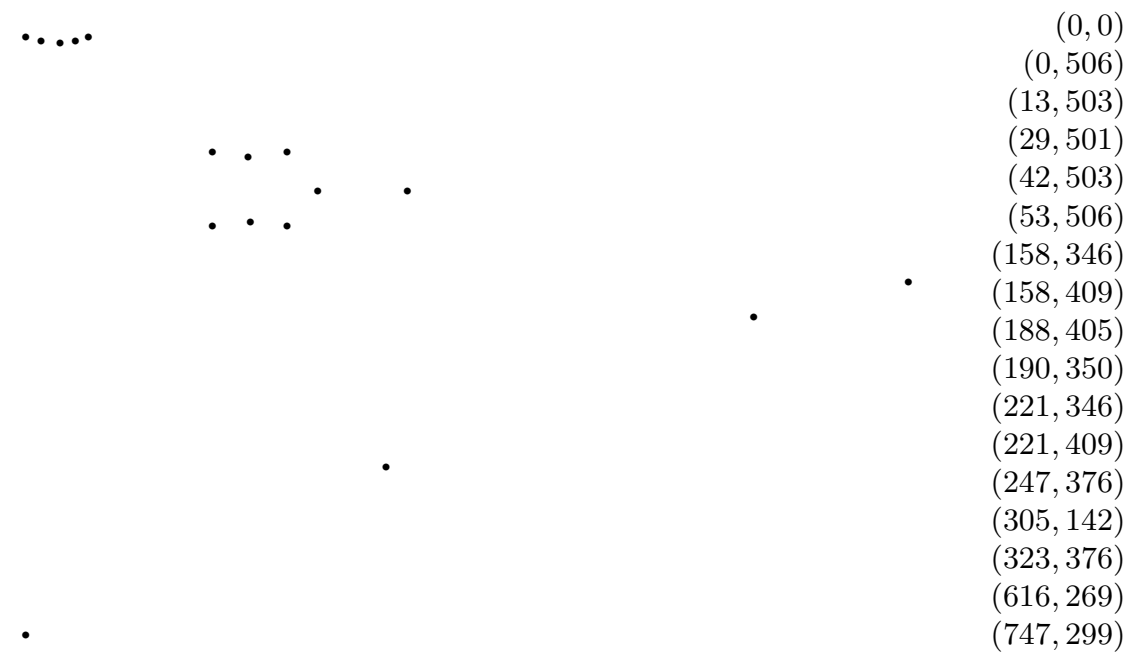

Рис. 1. Пример множества из 17 точек, не содержащего выпуклого шестиугольника не более, чем с одной точкой внутри

2. Краткое описание вычислений. Множество всех расположений конечного множества точек на плоскости имеют мощность континуума, поэтому к нему полный перебор не применим и необходимо предложить другое решение.

Для удобства будем считать, что все точки в рассматриваемых нами множествах имеют различные координаты по оси $x$. Для каждой тройки точек можно определить следуют ли они в порядке возрастания номеров по или против часовой стрелки. Сопоставим всем тройкам по одному биту, указывающему ориентацию. Полученный битовый вектор (для 17 точек он имеет длину $\left(\begin{array}{c}17 \\ 3\end{array}\right)=680$, для $18-\left(\begin{array}{c}18 \\ 3\end{array}\right)=816$ ) будем называть сигнатурой. Ясно, что имея сигнатуру можно найти все выпуклые многоугольники в исходном точечном множестве и количество точек внутри каждого из них. Например, условие наличия выпуклого шестиугольника задается равенством четырех бит сигнатуры нужным значениям. Тем самым, если перебрать все сигнатуры, которых конечное число, и убедиться в наличии нужного подмножества в каждой из них, то обе теоремы будут доказаны. Тем не менее, мощность множества всех сигнатур очень велика.

Предложенный алгоритм производит исчерпъвающий поиск такой сигнатуры, для которой соответствующее ей точечное множество не содержит нужного шестиугольника (т.е. пытается найти контрпример к теореме). Если к моменту завершения работы алгоритма не будет найдено ни одной, то обе теоремы будут обоснованы. Алгоритм последовательно заполняет в определенном порядке битовый массив, вплоть до присвоения значений всем элементам, т.е. до постороения полной сигнатуры. Заметим, что если массив заполнен еще не до конца, то это не мешает проверять условия наличия выпуклых шестиугольников, а также некоторые элементарные геометрические условия (они определяют возможность существования точечного множества с соответствующей сигнатурой) только для заполненных элементов. Если в частично заполненном массиве уже присутствуют значения, определяющие наличие нужного выпуклого шестиугольника, то дальнейшее его заполнение не происходит.

Укажем еще одну интересную особенность алгоритма. Заполнение битового массива на самом деле прекращается тогда, когда будут заполнены все специальным образом выбранные заранее элементы (их 139 для теоремы 1 и 140 для теоремы 2). После этого к ним применяются специальные проверки, называемые "однобитовая" и “двухбитовая", позволя- 
ющие отсеять те заполнения, которые заведомо не достроятся до сигнатуры, которая могла бы соответствовать точечному множеству, дающему контрпример к теореме (см. [8]). Авторы первоначальной версии алгоритма в своей статье [8] приводят следующие результаты для теоремы $g(6)=17$ : частично заполненных массивов получилось 20312212, после однобитовой проверки их осталось 23339, после двухбитовой - ни одного. Приведем такие же результаты для теоремы 1: частично заполненных массивов - 19698264093, после однобитовой проверки осталось 824389378 , после двухбитовой - ни одного. И для теоремы 2 : всего 26665261949, после однобитовой - 1836007604, после двухбитовой - ни одного.

\section{СПИСОК ЦИТИРОВАННОЙ ЛИТЕРАТУРЫ}

20 [1] P. Erdős, G. Szekeres, Compositio Math., 2 (1935), 463-470. [2] P. Erdős, G. Szekeres, Ann. Univ. Sci. Budapest. Eötvös Sect. Math., 3-4 (1961), 53-62. [3] P. Erdős, Austral. Math. Soc. Gaz., 5:2 (1978), 52-54. [4] W. Morris, V. Soltan, Bull. Amer. Math. Soc. (N.S.), 37:4 (2000), 437-458. [5] F. P. Ramsey, Proc. London Math. Soc. (2), 30 (1930), 264-286. [6] R. L. Graham, B. L. Rothschild, J. H. Spencer, Ramsey Theory, Wiley-Intersci. Ser. Discrete Math. Optim., John Wiley \& Sons, New York, 1990. [7] М. Холл, Комбинаторика, Мир, М., 1970. [8] G. Szekeres, L. Peters, ANZIAM J., 48:2 (2006), 151-164. [9] В. А. Кошелев, Докл. РАН, 415:6 (2007), 734-736. [10] Б. Х. Сендов, Фундамент. и прикл. матем., 1:2 (1995), 491-516. [11] J. D. Horton, Canad. Math. Bull., 26:4 (1983), 482-484. [12] H. Nyklová, Studia Sci. Math. Hungar., 40:3 (2003), 269-286. [13] В. А. Кошелев, Матем. заметки (в печати). [14] V.A. Koshelev, On Erdös-Szekeres problem and related problems, arXiv: math.CO/0910.2700. [15] В. А. Кошелев, Фундамент. и прикл. матем., 14:6 (2008), 91-120.

\section{В. А. Кошелев}

Поступило

Московский физико-технический институт

19.04.2010

(государственный университет)

E-mail: koshelev@mccme.ru 